\title{
Sterol-based oleogels' characterization envisioning food applications
}

\author{
Artur J Martins, ${ }^{\mathrm{a}, \mathrm{b}^{*}} \odot$ Miguel A Cerqueira, ${ }^{\mathrm{b}}$ Lorenzo M Pastrana, \\ Rosiane L Cunha ${ }^{c}$ and António A Vicente ${ }^{a}$
}

\begin{abstract}
BACKGROUND: Phytosterols, in particular a mixture of pure $\gamma$-oryzanol and $\beta$-sitosterol, develop a tubular system that is able to structure oil. In this study, different concentrations of a combination of $\gamma$-oryzanol and a commercial phytosterol mixture, Vitaesterol ${ }^{\circledR}$, were used for the development of edible oil oleogels.

RESULTS: Small-angle X-ray scattering (SAXS) and X-ray diffraction (XRD) were used to characterize at nano and molecular scale the aforementioned oleogels and confirm the formation of sterols-based hollow tubule structures. Increased hardness was observed with the increase of gelator content used in oleogel manufacturing. The produced oleogels showed promising features such as tailored mechanical strength and low opacity, which are important features when considering their incorporation into food products.

CONCLUSION: Despite differences in gel strength, oleogels exhibited textural characteristics that make these structures suitable for incorporation in food products. The oil migration profile associated with these oleogels can provide a solution for the controlled release of lipophilic compounds as well as for the retention of oil in cooked food products.

๔ 2018 Society of Chemical Industry
\end{abstract}

Keywords: oleogelation; oryzanol; phytosterols; self-assembly; oleogel

\section{INTRODUCTION}

Self-assembly of molecules presents itself as a very attractive mechanism to create new structures. One of the main challenges is to apply these structures in the production of novel food products. Understanding such mechanisms will help the exploration and explain how new materials can be used for the development of innovative products and applications. ${ }^{1}$ Interesting applications have been recently discussed regarding the use of oleogels in food formulations. The selection of an adequate combination of gelators, with distinctive oil gelling behaviour, is one of the most important steps in the process of the formulation of oleogels. ${ }^{2-10}$ One of the most relevant issues is to guarantee that gelation is achieved with the lowest possible amount of gelator, so that the oleogel effect is heightened, the cost of development is reduced and the final food production cost is maintained. In this way, oryzanol/phytosterol-based oleogels are among the most interesting ones not only because of the transparency and mechanical features with resemblance to a block of fat, when fully structured, ${ }^{11,12}$ but also because of their health-related properties. ${ }^{13} \gamma$-Oryzanol is a sitosterol ester with ferulic acid in its composition and responsible for lower intestinal cholesterol absorption, being pivotal elements for faecal removal of cholesterol. ${ }^{14-16}$ For the production of $\gamma$-oryzanol-based oleogels at room temperature, with the visual and mechanical characteristics mentioned earlier, the inclusion of phytosterol molecules is needed in order to promote multi-component oleogelation. In the literature an increase of the $\gamma$-oryzanol $/ \beta$-sitosterol ratio is described to lead to the formation of more transparent oleogels, the reverse being observed when the ratio is reduced. ${ }^{11,12}$ All phytosterols molecules have a steroidal ring base, a hydroxyl C-3 group, and a double bond that is placed between C-5 and C- 6 in the B-ring; these connection-sites must be available so that phytosterols are able to form a gel as a result of their interaction with $\gamma$-oryzanol. ${ }^{1,17}$ Translating to a molecular scale, $\gamma$-oryzanol and $\beta$-sitosterol assemble through the staking of the two molecules after being fully solubilized in an organic phase. This process is characterized by the proper cropping of the sitosterol units on top of each other during the cooling stage, leaving the ferulic acid moiety of the $\gamma$-oryzanol outside of the connected architecture. ${ }^{11}$ A recent study on the molecular dynamic modelling of this system demonstrated that the phytosterol's sterane group is stacked with oryzanol's sterane group. A hydrogen bond is responsible for the connection between both units and is formed between the hydroxyl group of the phytosterol and the carbonyl group of the oryzanol. ${ }^{18}$ The assembly between $\gamma$-oryzanol and $\beta$-sitosterol is characterized by a hollow

\footnotetext{
* Correspondence to: AJ Martins, CEB, Centre of Biological Engineering, University of Minho, Campus de Gualtar, 4710-0577 Braga, Portugal. E-mail:amartins@ceb.uminho.pt

a CEB, Centre of Biological Engineering, University of Minho, Braga, Portugal

b Department of Life Sciences, International Iberian Nanotechnology Laboratory, Braga, Portugal

c Department of Food Engineering, Faculty of Food Engineering, University of Campinas, UNICAMP, Campinas, Brazil
} 
tubular-shaped arrangement, eventually leading to gelation. This arrangement is formed due to the presence of the rigid four-rings system of sterol molecules as part of the fibrilar skeleton and of the ferulic acid moiety and its acyl-chain, which is more flexible and thus remains in the external surface, keeping the fibres apart. ${ }^{10}$ It is important to stress that if one of those components is not present, self-assembly will not happen and the hollow fibres would not be produced. Both components alone are able to form crystalline conformations. ${ }^{1,19}$ However, these conformations do not display nano-sized hollow tubules capable of gelling oil. ${ }^{20}$

Organoleptic characteristics of food products are often influenced by their textural and optical properties, which greatly affect the acceptance and preference by consumers. This has direct implications on the use of new ingredients, since such characteristics are affected by the used materials, processing conditions and storage. Oleogels are good examples of such ingredients, once their mechanical and optical properties change according to their formulation, as well as with the processing conditions. Oleogels may be incorporated in foods aiming at fat replacement but for this kind of application the textural properties should be strictly controlled.

This work has been performed for the first time on a commercial mixture of phytosterols, in order to achieve gelation in conjunction with, $\gamma$-oryzanol. In contrast with other reported works, the phytosterols used have only $\sim 45 \%$ of $\beta$-sitosterol (being this the major component) exposing the gelation mechanism to an increased amount of other phytosterols that can interfere in the co-assembly with $\gamma$-oryzanol. Despite the ability for other phytosterols to interact with $\gamma$-oryzanol to successfully develop oleogels, the structural and visual properties of these gels can differ. ${ }^{19}$ High oleic sunflower oil, a long-chain triglyceride (LCT), was used as the oil phase and the ability to form solid-like gels with reduced concentration of gelators $(6 \%, 8 \%$ and $10 \% \mathrm{w} / \mathrm{w})$ was evaluated. Our intention is to demonstrate the tailoring abilities in the production of these oleogel structures at macro and molecular levels, in particular how textural behaviour is directly associated to oil retention capacity, using lower amount and low-cost ingredients. This is surely relevant when evaluating the possibility of incorporating an oil structure in foods without compromising the final functionality and with the smallest possible influence on price. This work thus, aimed at the development of sterol-based oleogels using concentrations of gelators not exceeding $10 \% \mathrm{w} / \mathrm{w}$ and exploiting the gelling behaviour under steady-state conditions of a commercial mixture of phytosterol compounds (Vitaesterol $\left.{ }^{\circledR}\right)$, instead of pure $\beta$-sitosterol. The morphology of these oleogels was studied using polarized microscopy, small-angle X-ray scattering (SAXS) and X-ray diffraction (XRD). Texture measurements allowed gel hardness, final force and adhesiveness to be determined. Oil migration and colour were also evaluated for increasing concentrations of sterol mixtures.

\section{MATERIALS AND METHODS}

\section{Raw materials and oleogels development}

High oleic sunflower oil fraction composed by: $0.1 \%$ of C14:0, $3.8 \%$ of $\mathrm{C} 16: 0,3.3 \%$ of $\mathrm{C} 18: 0,80.1 \%$ of $\mathrm{C} 18: 1,10.7$ of $\mathrm{C} 18: 2,0.3 \%$ of $\mathrm{C} 18: 3,0.4 \%$ of $\mathrm{C} 20: 0$ and $0.1 \%$ of $\mathrm{C} 20: 1$ was kindly provided by Cargill (São Paulo, Brazil). $\gamma$-Oryzanol was purchased from Oryza Co. (Ichinomiya, Japan) and the mixture of phytosterols Vitaesterol ${ }^{\circledR}$, was kindly provided by Vitae Naturals (Toledo, Spain). This phytosterol mixture is composed by $\beta$-sitosterol ( 45\%), campesterol $(-25 \%)$, stigmaesterol $(-23 \%)$, brassicasterol $(\sim 1 \%)$, other sterols $(\sim 3 \%)$.
The 1:1 ratio of oryzanol/sitosterol was reported as the ratio that allows the firmest transparent gel to be obtained. ${ }^{12}$ However in line with other previous works in the field, we developed oleogels using the ratio of $60: 40(w / w)$ oryzanol/phytosterol in order to understand the effect of a reduced concentration of $\beta$-sitosterol $(\sim 45 \%)$, targeting sterol-based gels production. Different concentrations were tested in order to determine the critical concentration of gelation (results not shown); sterol-oleogels (STOs) with less than $6 \%(w / w)$ of total gelators concentration were not self-sustained. Different concentrations of this mixture of gelators were used to produce sterol-based oleogels: $6 \%, 8 \%$ and $10 \%$ $(w / w)$ - STO6, STO8 and STO10, respectively. All gels were produced by heating the mixture of oil phase and gelators at $80^{\circ} \mathrm{C}$ for $30 \mathrm{~min}$, under magnetic stirring to ensure full solubilization of the compounds in the oil phase prior to the cooling stage. The samples were left cooling at room temperature and then stored at $4{ }^{\circ} \mathrm{C}$ for at least $24 \mathrm{~h}$. Before analyses, the samples were left again at room temperature for $24 \mathrm{~h}$.

\section{Polarized microscopy and segmentation}

Sterol-based oleogels were cut and placed in a cover slip and analysed at room temperature with a polarized light microscope (Olympus System Microscope model BX51TF, Olympus America Inc., Center Valley, PA, USA) equipped with a digital camera (Olympus EX300, Olympus America Inc.). Pictures were taken at a magnification of $120 \times$ and $320 \times$. Images were segmented using the threshold colour plugin available in ImageJ software. This plugin enables an image segmentation in distinctive black and white colour areas defined using an automatically calculated threshold. ${ }^{21}$

\section{Small-angle X-ray scattering (SAXS) and X-ray diffraction (XRD)}

All SAXS analyses were performed at room temperature, using the beamline of the National Synchrotron Light Laboratory (LNLS, Campinas, Brazil). The apparatus is equipped with an asymmetrically cut and bent silicon (1:1:1) monochromator that yields a monochromatic $(\lambda=1.54 \AA)$ and horizontally focused beam. In order to record SAXS intensity a position-sensitive X-ray detector and a multichannel analyser were used. The I(q) was evaluated as a function of the modulus of scattering vector $q=(4 \pi / \lambda) \sin (\theta / 2)(\theta$ being the scattering angle). Each SAXS pattern corresponds to a data collection time of $100 \mathrm{~s}$ in the range of $0.119 \mathrm{~nm}^{-1}<q<4.811 \mathrm{~nm}^{-1}$. Scattering data was corrected by subtraction of the pure mica and sampler signal. The determination of the crystal structure was done using Bragg's law, through the relative position of the diffraction peaks. The lattice parameter $d$ (periodicity) of structures was obtained from the position $q^{*}$ of the first (and most intense) diffraction peak. The type of crystal organization and crystal structure was evaluated, being the evaluation performed by the Guinier plot, where through the $\log -\log$ plot ( versus q) and the determination of the slope at small angles the type of structure (e.g. flat or rod-type structure) can be determined. ATSAS software was used to gather SAXS data, then PRIMUS (Mac version 2.8.3) software was used to analyse the data (with program BODIES), making it possible to determine the expected radius of gyration $\left(R_{g}\right)$ associated with the hollow tubular structure that is reported for this type of self-assembled structure. XRD was employed to understand the differences between oleogels at molecular length scales. Crystal polymorphic structure 
was evaluated by scattering methodology with an X-Ray Diffractometer X Pert PRO MRD system from PanAnalytical. PANanalytical $X^{\prime}$ Pert HighScore Plus was the software used to gather data and analyse peak diffractions. The XRD diffractograms were acquired at room temperature, angular scans from $5^{\circ}$ to $50^{\circ}(2 \theta)$ were performed with a Cu source, $\mathrm{X}$-ray tube $(\lambda=1.54056 \AA)$ at $45 \mathrm{kV}$ and $40 \mathrm{~mA}$. The fine calibration offset for $2 \theta=-0.0372^{\circ}$.

\section{Textural analysis}

The textural parameters of sterol-based oleogels were measured with a Texture Analyser TA-HD Plus (Stable Microsystems, Godalming, UK). Samples were prepared (approximately $3 \mathrm{~g}$ ) in plastic cups and the tests were performed using a $10 \mathrm{~mm}$ cylindrical probe. The measured force in compression was made with a test speed of $1 \mathrm{~mm} \mathrm{~s}^{-1}$, and then the probe was pulled out of the sample at $10 \mathrm{~mm} \mathrm{~s}^{-1}$. This experiment allowed values of firmness (force), final force (value recorded at the endpoint of the probe travel) and adhesivity (recorded in the dragging final stage) to be determined. The data were calculated using Texture Exponent v.6.1.1.0 software by Stable Microsystems.

\section{Optical properties}

Colour and opacity of oleogels were evaluated using a Minolta colorimeter (CR 300; Minolta, Tokyo, Japan). Each mean is the result of five measurements in one triplicate oleogel sample. A white standard colour plate was used for background measurements and also for the calibration of the instrument $(Y=493.5, x=40.3114, y=40.3190)$. Then CIE $L^{*} a^{*} b^{*}$ values of each sample were evaluated by reflectance measurements. The opacity of each oleogel was calculated from reflectance measurements and corresponds to how much light passes through it. ${ }^{22}$ The higher this value is, the lower the amount of light that can pass through the material. This parameter was calculated according to the Hunter lab method, as the relationship between the opacity of each sample on a black standard $\left(Y_{b}\right)$ and the opacity of each sample on a white standard $\left(Y_{\mathrm{w}}\right)$.

\section{Oil migration}

Oleogel oil migration (OMG) profile was measured following the methodology described by Dibildox-Alvarado et al. ${ }^{23}$ with some modification. Samples were prepared cutting a disc of $15 \mathrm{~mm}$ diameter and $3.5 \mathrm{~mm}$ of thickness. After that, each one of the discs was placed on top of a round filter paper (Whatman \#1, $90 \mathrm{~mm}$ diameter) and then incubated at $20^{\circ} \mathrm{C}$. Collected data is a result of five replicates for each oleogel. The weight of the filter papers was determined during the first $8 \mathrm{~h}$ and then every day until a final period of $96 \mathrm{~h}$. The weight of a 'blank' filter paper was measured in all experiments. The percent of the amount of oil absorbed from each one of the samples was calculated using Eqn (1).

$$
O M G=\left[\frac{(\Delta \text { paper weight })-(\Delta \text { blank weight })}{\text { paper weight }(t=0)}\right] \times 100
$$

\section{Statistical analysis}

All experiments subjected to statistical analysis were carried out in quintuplicate. The results were reported as an average and standard deviation of these measurements. The statistical analyses were performed using analysis of variance, Tukey's mean comparison test $(P<0.05)$ (GraphPad Software, Inc., La Jolla, (A, USA).

\section{RESULTS AND DISCUSSION}

\section{Polarized microscopy and segmentation}

The use of polarization during microscopy is one of the most efficient techniques to identify the presence of crystalline structures in edible oils. Polarized microscopy allows the identification of the crystalline structures that result from the gelation of the oryzanol and phytosterols in the organic solvent. The structures observed through microscopic lenses are the result of the larger amounts of phytosterol. The observed crystals can be explained by the phytosterols (it is expected that only $\beta$-sitosterol interacts with oryzanol) that are not able to interact with oryzanol to form the hollow-tubules that characterizes this sterol system. The haziness of these gels can be a result of the sterol mixture, which includes no more than $45 \%$ of $\beta$-sitosterol. Owing to the enlarged amount of other phytosterols, some disparities in the already reported molecular aggregation processes might have happened. Figure 1(A), (B) and (E) show the polarized microscopy images of STO6, STO8 and STO10, respectively (magnification $120 \times)$. Figure $1(C)$ and (D) were taken from Fig. 1(A) and (B) with
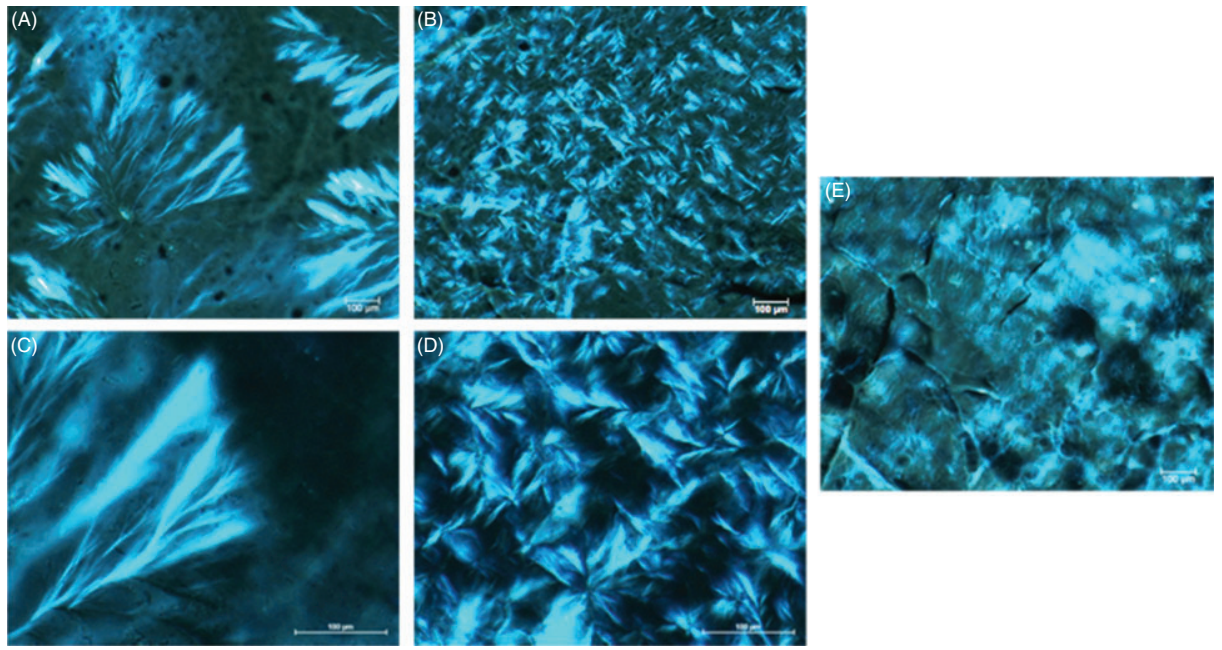

Figure 1. Polarized micrographs of sterol-based oleogels. (A), (B) and (E) correspond to STO6, STO8 and STO10, respectively. (C), (D) correspond to (A) and (B), respectively, with larger magnification. 


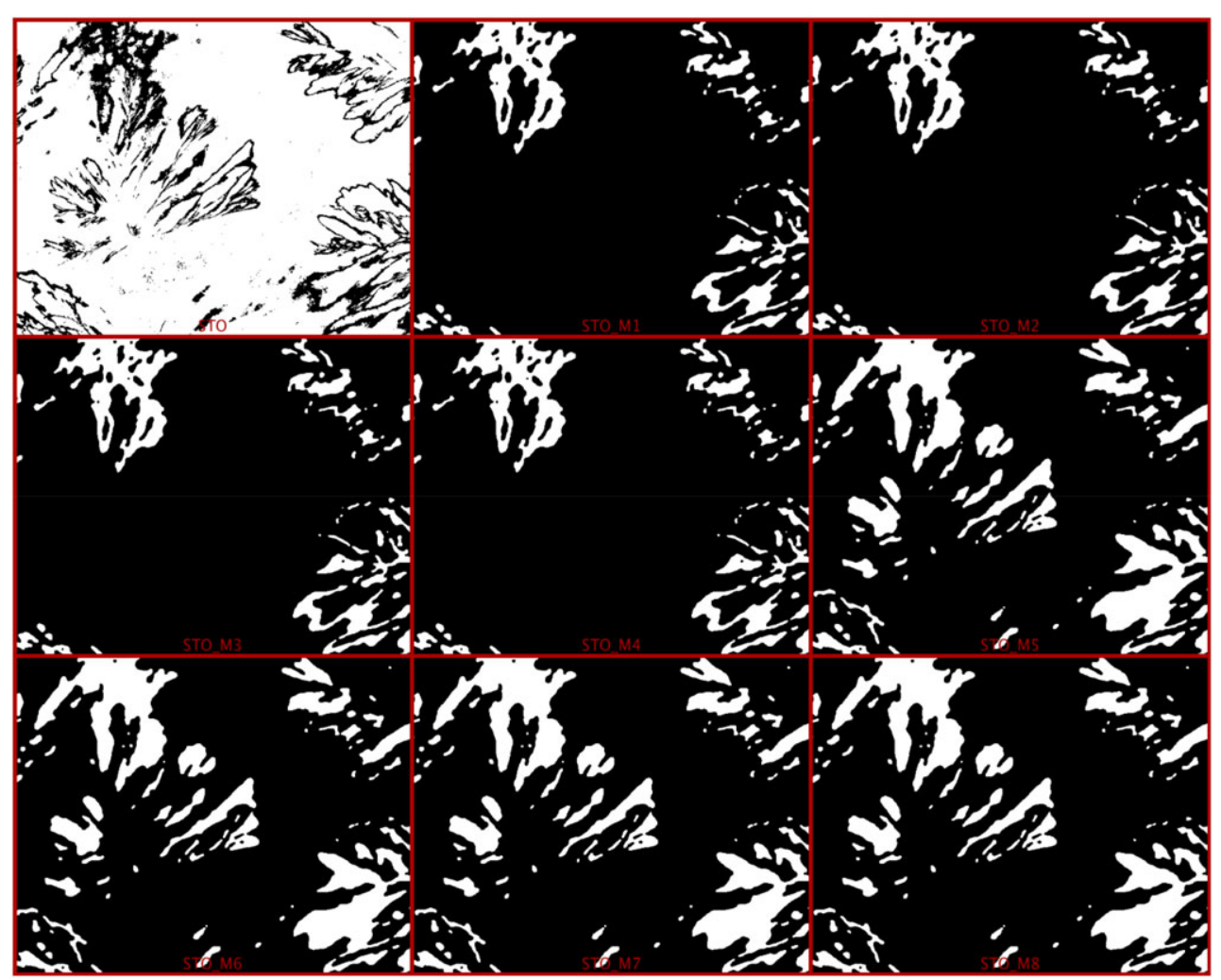

Figure 2. Processing and consequent segmentation of STO6 polarized micrograph. The segmentation was performed using the Mixed Segmentation Algorithm.

larger magnification (320x). For STO6 the branching of these crystals is clear and they are very well distinguished. These structures when formed will also contribute to the entrapment of the oil phase. It is possible to identify a large amount of structures as the concentration of gelators is increased, but it is more difficult to fully distinguish these structures. For the highest concentration of structurant (10\%) it was not possible to identify the single crystal structures (Fig. 1(E)). This may happen because of the presence of crystals in different morphological planes inside the oleogel structure. With polarizer shifting and consequent light blocking, it was possible to direct the angle at which light reaches the sample in order to generate contrast with the background, thus allowing a better visualization of crystals in the gelators in the oil phase. We also hypothesize that the presence of several sterol components is responsible for the haziness (and material agglomeration) on the formed oleogels. This also justifies the capacity to identify the crystal structures in polarized microscopy in the oleogel with 60:40 ratio of oryzanol and sitosterol; according to the literature this ratio will produce (with pure components) glossy and transparent gels, which is not the case here.

Figure 2 represents the segmentation of Fig. 1(A) using Image J software. This treatment allows the different geographical planes to be recognized where the crystals are disposed in the STO samples and thus how the deposition or stacking is responsible for the self-sustainment of the oleogels. Furthermore, the recognition of the crystals is clearer against the dark background, and therefore less dense crystalline material is observed. This less dense crystalline material consequently will affect the gel opacity and will be involved in gel textural characteristics, as is discussed later. This provides a good overview of the crystalline fibrilar deposition in the oleogel bulk structure. The use of the micrograph of STO6, which is representative of the lower concentration of gelators used, allowed the best segmentation fitting to be obtained. This happens because the crystals density is the lowest of all STOs concentration tested, which facilitates the task of discerning the crystal disposition without much background noise. In fact, it is very difficult to understand this if the segmentation is performed using the images of oleogels for higher concentrations of sterols because of the more crowded background.

\section{Small-angle X-ray scattering (SAXS) and X-ray diffraction (XRD)}

Figure 3(A) shows the SAXS spectra for STO6, STO8 and STO10 samples. The spectra show that for higher concentrations of gelator there is an increase of the scattering intensity for the observed peaks. From the log-log plot ( versus q) we conclude that the obtained structures are hollow cylinders, being in agreement with the literature. The reports, mentioned earlier, show that the maxima decrease, regarding interference peaks, is proportional to $q^{-2}$, while for solid cylinders the model would require a decrease proportional to $q^{-4} .^{12}$ Studies with similar geometric structures (hollow tubules) formed by amphiphilic peptides are reported to have also scattering curves with $q^{-2}$ at low $q$ values. ${ }^{24}$ Three major reflections are observed for all STOs, being the lattice of the crystalline arrangements at 1.1 (001), $2.01(002)$ and $2.75 \mathrm{~nm}^{-1}$ (003). These values deviate from the values presented in the literature. ${ }^{4,12}$ Such a change (namely in the first reflection) can be explained by the significant amount of other phytosterols present in the mixture, leading to a shift of the peak from 0.99 to $1.10 \mathrm{~nm}^{-1}$. Despite that, the broad pattern of self-assembled tubules and the presented reflections are in line with the ones presented by Adel 

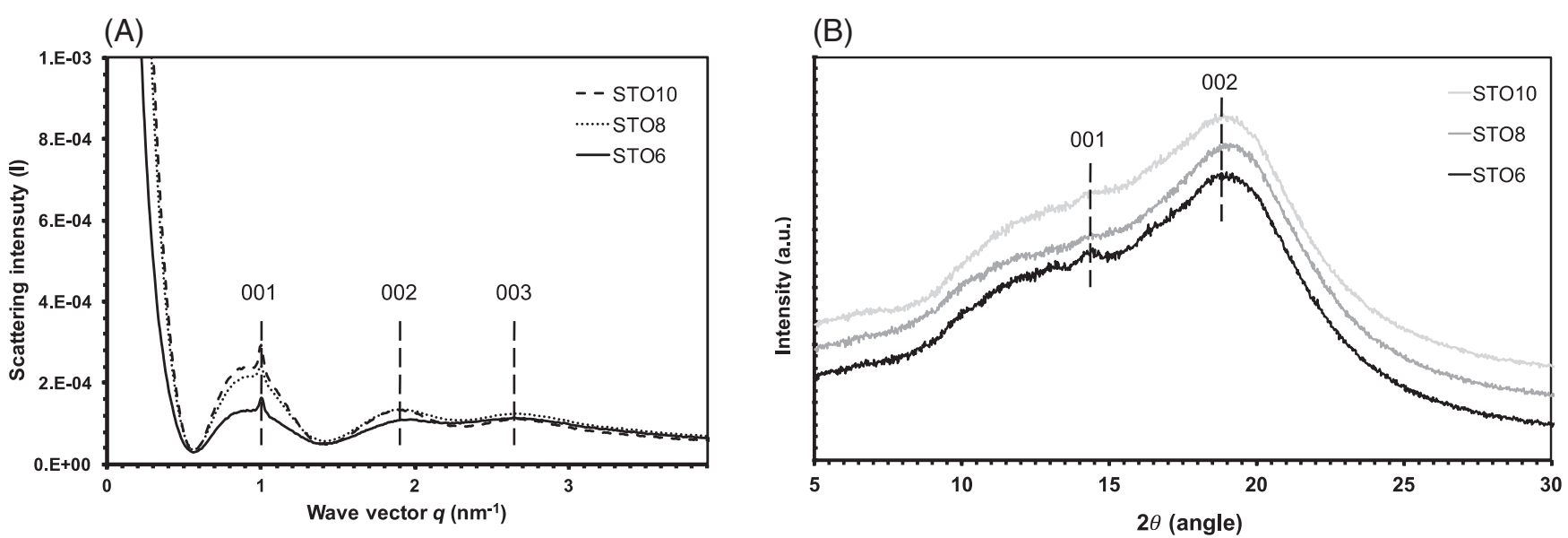

Figure 3. (A) Small-angle X-ray scattering (SAXS) pattern for STO10, STO8 and STO6. (B) X-ray diffraction (XRD) spectra for STO10, STO8 and STO6.

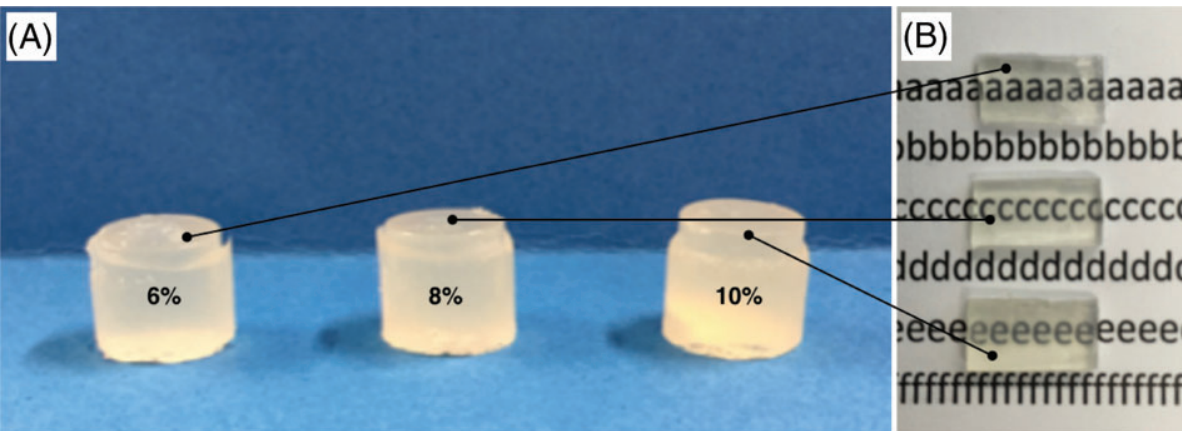

Figure 4. (A) Visual appearance of STO10, STO8 and STO6. (B) Transparency associated with these type of oleogels.

et al. ${ }^{25}$ The $R_{g}$ parameter was obtained using the hollow-cylinder geometry as the predefinition in Primus Software. This parameter was obtained from the Guinier region of the full SAXS spectra, and gives information on the radius of spherical-like structures or ensembles. ${ }^{26}$ Lower amounts of $\beta$-sitosterol did not prevent the formation of nano-scale tubules, formed by phytosterol interaction with $\gamma$-oryzanol. The expected $R_{g}$ values were very close for all concentrations, ranging between 5.08 and $5.68 \mathrm{~nm}$. This should be an indication that different concentrations of gelator are not responsible for changes in tubule thickness. However, different concentrations of gelators will influence the size and number of crystals.

Reducing the length-scale of analysis, XRD allowed the crystal structure of oleogels at the molecular scale to be evaluated. Figure $3(\mathrm{~B})$ presents $\mathrm{X}$-ray spectra of the STOs for the three tested concentrations, vertical shifts were applied to the curves to improve the observation. Spectra show a large amorphous peak $\left(10^{\circ}-15^{\circ}\right)$ which is evidence of a mixed crystalline phase occurring. ${ }^{17}$ In contrast to the patterns shown by the pure components, the crystalline pattern of the oleogel is not as clear. ${ }^{10}$ The peak associated with the oil phase (approximate angle of $18.9^{\circ}$ ) relates to a lattice value of $4.63 \pm 0.05 \AA$ (002), similar to the ones recorded for pure oryzanol/sitosterol oleogels with sunflower oil and medium-chain triglyceride (MCT)-based oleogels. ${ }^{11,27}$ It is possible to recognize that the crystallite monogram is not clear, however besides the main peak, we also identified some weaker reflections and we can associate the peaks at $d=6.18 \pm 0.07 \AA$ (001), in the mixed crystalline phase with $\beta$-sitosterol crystallites in oil. ${ }^{27}$ The presence of these weaker reflections in a very smooth

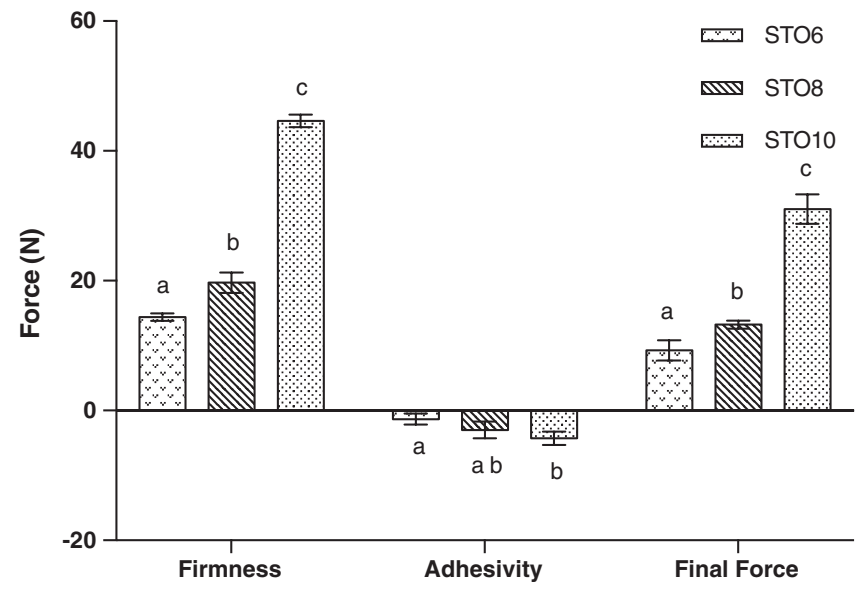

Figure 5. Texture parameters for STO samples. Different letters mean that samples are significantly different (for the same parameter measured).

pattern was also observed in the formation of tubules in the mixture of oryzanol + phytosterols (tall oil sterol from Unilever with $78.1 \% \beta$-sitosterol, $10.3 \%$ campesterol) $16 \%$ total mass in refined sunflower oil. ${ }^{25}$

\section{Texture analysis}

STOs were self-standing (Fig. 4(A)), maintaining their structure after hand compression and showed transparency (Fig. 4(B)), as a result of the oil-phase colour and submicron structures formed during gelation. Figure 5 presents the results of texture parameters 
Table 1. Colour parameters of oleogel samples

\begin{tabular}{|c|c|c|c|c|c|c|c|c|c|}
\hline & \multicolumn{3}{|c|}{ STO6 } & \multicolumn{3}{|c|}{ STO8 } & \multicolumn{3}{|c|}{ STO10 } \\
\hline & Mean & SD & $n$ & Mean & SD & $n$ & Mean & SD & $n$ \\
\hline Opacity & $12.4^{a}$ & 0.26 & 3 & $13.81^{b}$ & 0.11 & 3 & $13.91^{b}$ & 0.28 & 3 \\
\hline$L^{*}$ & $90.451^{a}$ & 0.721 & 3 & $87.554^{b}$ & 0.339 & 3 & $87.127^{b}$ & 0.289 & 3 \\
\hline$a^{*}$ & $-0.499^{a}$ & 0.057 & 3 & $-0.443^{a}$ & 0.003 & 3 & $-0.58^{a}$ & 0.069 & 3 \\
\hline$b^{*}$ & $7.555^{a}$ & 0.921 & 3 & $10.299^{b}$ & 1.148 & 3 & $11.815^{c}$ & 0.701 & 3 \\
\hline
\end{tabular}

Note: Different letters in the same row correspond to statistically different samples for a $95 \%$ confidence level. SD, standard deviation; $n$, number of replicates.

determined for STO6, STO8 and STO10. It shows that an increase in the concentration of gelators leads to an increase in the force the samples can withstand (force peak and final force) and adhesivity, being higher for oleogels with $10 \%(w / w)$ of gelators. The obtained values for firmness/hardness (force peak) and final force were significantly different $(P<0.05)$ for all concentrations, while for adhesivity the values between STO6 and STO10 are the only ones that present statistically significant differences. The final force is the value recorded at the endpoint of the travelling probe, and these results show the strength of the gel structure after the compression test. The strongest oleogel (STO10) presented values of force peak and final force which were two-fold higher than the values for the lower concentrations (6\% and $8 \%$ ). These results are explained by a significantly stronger crystal network that is formed using a higher gelator concentration. This fact is well displayed in the polarized micrographs. The oleogels with a more interconnected crystal network showed higher mechanical properties, as can be observed in textural data analyses. These features can be important when such oleogels are used in foods, so that certain textural behaviours can be mimicked according to the oleogel formulation. For example, sausages with high fat content present firmness values of approximately $12.20 \mathrm{~N}^{28}$ which are of the same order of magnitude of the values obtained in the present work (Fig. 4).

\section{Optical properties}

All oleogels were shown to be quite transparent with a very slight yellowish tonality (Fig. 4). Table 1 presents the data regarding colour parameters, namely $L^{*}$ (lightness), $a^{*}$ (range between green and magenta) and $b^{*}$ (range between blue and yellow). Regarding these data, the lightness values $\left(L^{*}\right)$ record statistically significant differences between STO6 oleogels and the other two, being in all cases relatively high values, ranging from 87.13 to 90.45 . Also for $b^{*}$ values, the results confirm the visual observation that a slight yellowish coloration is identifiable in all samples, possibly resulting from the coloration of sunflower oil, which also increases with the increase of sterols concentration.

It is important to notice that the samples with the highest concentration of sterols (STO10), still exhibit high values of transparency, which can be a very desirable feature for an ingredient aimed at being incorporated in food products. The greater the value associated to opacity, the lower is the transparency of such sample. Table 1 shows that the presence of larger amounts of sterols is responsible for an increase of opacity with significant differences between STO6 and the other two types of STO (STO8 and STO10, between which no statistically significant differences were observed). These opacity results are in line with the crystalline network that was observed in the polarized microscopic analyses. As seen in the polarized micrographs (Fig. 1), the density of the crystal structures formed in STO8 and STO10 was much higher than the one observed for STO6.

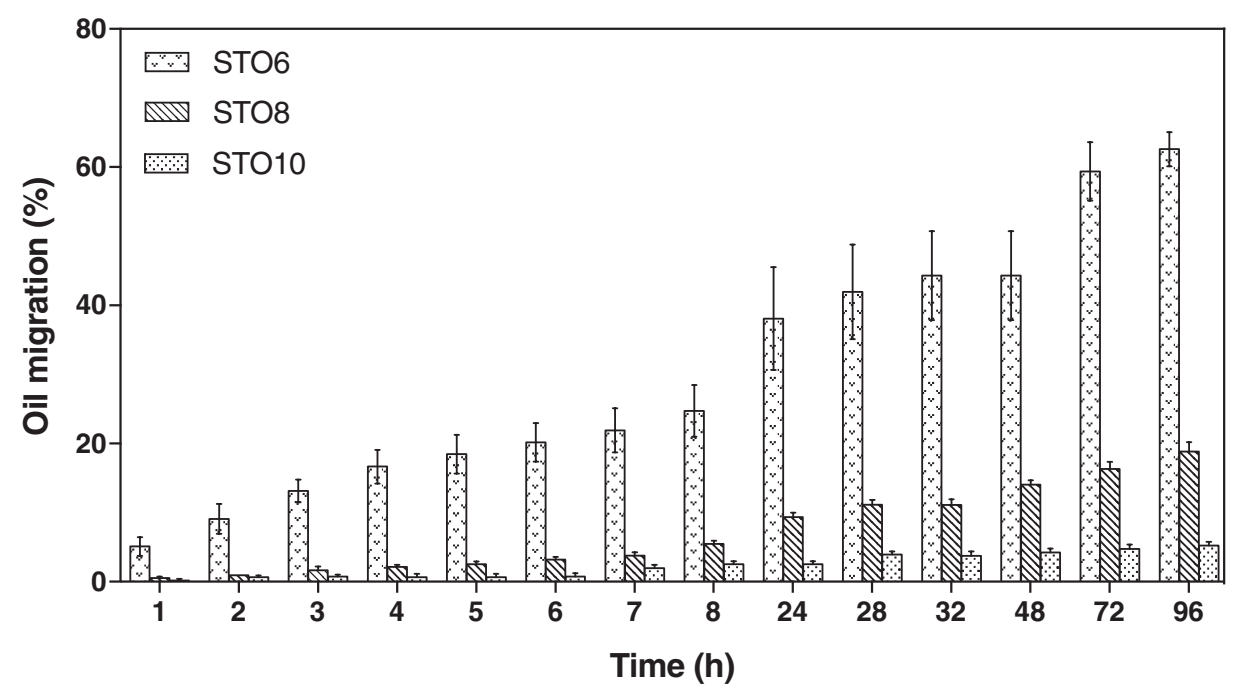

Figure 6. Oil migration (\%) of STOs during $96 \mathrm{~h}$. 


\section{Oil migration}

Figure 6 presents the results of oil migration (OMG) for each STO through time. STO10 samples present the lowest value of OMG. This can be explained by the increased amount of crystalline structures (lower porosity) observed by polarized microscopy, which also led to a higher hardness exhibited by this particular formulation. For STO10 samples the OMG profile stabilized after $24 \mathrm{~h}$, being stable until the end of the experiment (96h). The STO6 samples revealed to be less stable once oil migration occurs continuously until the end of the experiment. STO8 gels showed an intermediate behaviour. However, as previously observed for other parameters, the most significant change was observed when the concentration of the gelator increased from $6 \%$ to $8 \%(w / w)$. This is justified by the proximity to the critical point of gelation (as already mentioned, slightly below $6 \%$ of the total sterols concentration) as gels with less available mass of gelator, and consequently less crystals can facilitate oil leakage at the surface.

These results show the capacity of STOs to block or reduce OMG in fat-based multi-component foods, but also opens the possibility of using STOs as delivery systems of lipophilic compounds. A previous study showed that in a multi-layer system, using gelled chocolate containing sterol-based oleogel in its composition, the level of oil migration to the chocolate layer was reduced by around $50 \%{ }^{29}$ Regarding statistical analyses, only the OMG values for STO10 for migration times of 24, 48, 72 and $96 \mathrm{~h}$ showed no statistically significant differences $(P<0.05)$.

\section{CONCLUSION}

Oleogels based in a commercial mixture of sterols were produced using different gelator concentrations and high-oleic sunflower oil as solvent. SAXS results confirmed the hollow fibrilar shape, characteristic of the interaction of these gelators and self-assembly. Scattering data and polarized microscopy allowed the understanding of how gelator arrangements are disposed in order to be able to entrap the oil and consequently induce gelation. Textural characteristics are modified (i.e. stronger oleogels) with the increase of gelator concentrations, showing the possibility of tailoring these structures to fit the requirements of the intended applications. The produced oleogels showed other promising features such as mechanical strength and good visual characteristics (low opacity values), important when considering the incorporation in food products. Oil migration was affected by the sterols concentration and related to the gel strengths. This is important for the application of these oleogels in foods, either as fat replacers or in the delivery of lipophilic bioactive compounds. Future work will comprise the formation of sterol-based oleogels without this commercial mixture in order to understand the crystallization phenomena and how different cooling temperatures will affect the gel characteristics.

\section{ACKNOWLEDGEMENTS}

Artur Martins is recipient of a fellowship supported by a doctoral advanced training (call NORTE-69-2015-2015) funded by the European Social Fund under the scope of Norte2020 - Programa Operacional Regional do Norte. The authors thank the Brazilian Synchrotron Light Laboratory (LNLS, Campinas, SP, Brazil) for the opportunity to carry out SAXS measurements. Artur Martins also thank the UNICAMP, FAEPEX INTERNACIONAL 2015 Project. This study was supported by the Portuguese Foundation for Science and Technology (FCT) under the scope of the strategic funding of UID/BIO/04469 unit and COMPETE 2020 (POCl-01-0145-FEDER-006684) and BioTecNorte operation (NORTE-01-0145-FEDER-000004) funded by the European Regional Development Fund under the scope of Norte2020 - Programa Operacional Regional do Norte.

\section{REFERENCES}

1 Rogers MA, Co-operative self-assembly of cholesterol and $\gamma$-oryzanol composite crystals. CrystEngComm 13:7049 (2011).

2 Martins AJ, Cerqueira MA, Cunha RL and Vicente AA, Fortified beeswax oleogels: effect of $\beta$-carotene on the gel structure and oxidative stability. Food Funct 8:4241-4250 (2017).

3 Martins AJ, Vicente AA, Cunha RL and Cerqueira MA, Edible oleogels: an opportunity for fat replacement in foods. Food Funct 9:758-773 (2018).

4 Bot A, den Adel R, Roijers EC and Regkos C, Effect of sterol type on structure of tubules in sterol $+\gamma$-oryzanol-based organogels. Food Biophys 4:266-272 (2009).

5 Co ED and Marangoni AG, Organogels: an alternative edible oil-structuring method. J Am Oil Chem Soc 89:749-780 (2012).

6 Patel AR and Dewettinck K, Edible oil structuring: an overview and recent updates. Food Funct 7:20-29 (2016).

7 Stortz TA and Marangoni AG, Ethylcellulose solvent substitution method of preparing heat resistant chocolate. Food Res Int 51:797-803 (2013).

8 Davidovich-Pinhas M, Barbut S and Marangoni AG, The gelation of oil using ethyl cellulose. Carbohydr Polym 117:869-878 (2015).

9 Mert B and Demirkesen I, Reducing saturated fat with oleogel/shortening blends in a baked product. Food Chem 199:809-816 (2016).

10 Calligaris S, Mirolo G, Da Pieve S, Arrighetti G and Nicoli MC, Effect of oil type on formation, structure and thermal properties of $\gamma$-oryzanol and $\beta$-sitosterol-based organogels. Food Biophys 9:69-75 (2014).

11 Bot A, Veldhuizen YSJ, den Adel R and Roijers EC, Non-TAG structuring of edible oils and emulsions. Food Hydrocoll 23:1184-1189 (2009).

12 Bot A, Den Adel R and Roijers EC, Fibrils of $\gamma$-oryzanol $+\beta$-sitosterol in edible oil organogels. J Am Oil Chem Soc 85:1127-1134 (2008).

13 Wang L, Lin Q, Yang T, Liang Y, Nie Y, Luo Y et al., Oryzanol modifies high fat diet-induced obesity, liver gene expression profile, and inflammation response in mice. J Agric Food Chem 65:8374-8385 (2017).

14 Vaikousi H, Lazaridou A, Biliaderis CG and Zawistowski J, Phase transitions, solubility, and crystallization kinetics of phytosterols and phytosterol-oil blends. J Agric Food Chem 55:1790-1798 (2007).

15 Sawalha H, Den Adel R, Venema P, Bot A, Flöter E and Van Der Linden $\mathrm{E}$, Organogel-emulsions with mixtures of $\beta$-sitosterol and Y-oryzanol: influence of water activity and type of oil phase on gelling capability. J Agric Food Chem 60:3462-3470 (2012).

16 Sawalha $H$, Venema $P$, Bot A, Flöter $E$ and van der Linden $E$, The influence of concentration and temperature on the formation of $\gamma$-oryzanol $+\beta$-sitosterol tubules in edible oil organogels. Food Biophys 6:20-25 (2011).

17 Alhasawi FM and Rogers MA, Ternary phase diagram of $\beta$-sitosterol- $\gamma$-oryzanol-canola oil. J Am Oil Chem Soc 90:1533-1540 (2013).

18 Dalkas G, Matheson AB, Vass H, Gromov A, Lloyd GO, Koutsos V etal., Molecular interactions behind the self-assembly and microstructure of mixed sterol organogels. Langmuir 34:8629-8638 (2018).

19 Bot A and WGM A, Structuring of edible oils by mixtures of $\gamma$-oryzanol with $\beta$-sitosterol or related phytosterols. J Am Oil Chem Soc 83:513-521 (2006).

20 Patel AR, A colloidal gel perspective for understanding oleogelation. Curr Opin Food Sci 15:1-7 (2017).

21 Hotaling NA, Bharti K, Kriel H and Simon CG Jr, DiameterJ: a validated open source nanofiber diameter measurement tool. Biomaterials 61:327-338 (2015). 
22 Casariego A, Souza BWS, Cerqueira MA, Teixeira JA, Cruz L, Diaz R et al., Chitosan/clay films' properties as affected by biopolymer and clay micro/nanoparticles' concentrations. Food Hydrocoll 23:1895-1902 (2009).

23 Dibildox-Alvarado E, Rodrigues JN, Gioielli LA, Toro-Vazquez JF and Marangoni AG, Effects of crystalline microstructure on oil migration in a semisolid fat matrix. Cryst Growth Des 4:731-736 (2004).

24 Bitton R, Schmidt J, Biesalski M, Tu R, Tirrell M and Bianco-Peled H, Self-assembly of model DNA-binding peptide amphiphiles. Langmuir 21:11888-11895 (2005).

25 Adel R, Heussen PCM and Bot A, Effect of water on self-assembled tubules in $\beta$-sitosterol $+\gamma$-oryzanol-based organogels. J Phys Conf Ser 247:12025 (2010).
26 Davidovich-Pinhas $M$, Barbut $S$ and Marangoni AG, Development, characterization, and utilization of food-grade polymer oleogels. Annu Rev Food Sci Technol 7:65-91 (2016).

27 Von Bonsdorff-nikander A, Karjalainen M, Rantanen J and Christiansen L, Physical stability of a microcrystalline b-sitosterol suspension in oil. Eur J Pharm Sci 19:173-179 (2003).

28 Petersson K, Godard O, Eliasson AC and Tornberg E, The effects of cereal additives in low-fat sausages and meatballs. Part 2: Rye bran, oat bran and barley fibre. Meat Sci 96:503-508 (2014).

29 Wendt A, Abraham K, Wernecke C, Pfeiffer J and Flöter E, Application of $\beta$-sitosterol $+\gamma$-oryzanol-structured organogel as migration barrier in filled chocolate products. J Am Oil Chem Soc 94:1131-1140 (2017). 